\title{
Análise do desenvolvimento de novos produtos no laboratório de uma Instituição de Ensino
}

\author{
Analysis of new products's development in the laboratory of an Educational Institution \\ Luize Nunes Dantas*1; Fernanda Francisquine Sant'Ana ${ }^{1}$; Fernando Monsores Almeida1; Luiz Felipe \\ Caramez Berteges $^{2}$
}

Como citar esse artigo. Dantas, L.N; Sant'Ana, F.F; Almeida, F.M; Berteges, L.F.C. Análise do desenvolvimento de novos produtos no laboratório de uma Instituição de Ensino. Revista Teccen. 2021 Jul./Dez.; 14 (2): 36-42.

\begin{abstract}
Resumo
A busca pela inovação e novas tecnologias de mercado cresceu ao longo dos anos, ocasionando o surgimento da $4^{\mathrm{a}}$ Revolução Industrial, caracterizada pela Indústria 4.0. Com isso, surgiu a necessidade de investimento, por parte das empresas e grandes organizações, em áreas relacionadas ao processo de desenvolvimento de produto (PDP), que consiste em um processo de negócio que formaliza as atividades para o desenvolvimento de produtos e serviços. A organização deste processo auxilia na melhoria da qualidade do produto e na redução de custos, fazendo com que a empresa se torne mais ágil e competitiva. O artigo objetiva-se em mapear, analisar e explorar os fluxos que ocorrem no processo de desenvolvimento de novos produtos em um laboratório de uma instituição de ensino. Como metodologia é aplicado o sistema de pesquisa qualitativa, descritiva e exploratória, embasada no presente estudo. Com o seguinte estudo foi possível entender a necessidade em formalizar, mapear e compreender o processo a fim de entendimento e comparação com a teoria. Pode-se concluir que o setor de P\&D vem ganhando espaço no mercado, e o investimento no processo de desenvolvimento de novos produtos é de extrema importância para toda e qualquer organização e que seu pleno conhecimento gera oportunidades de melhoria para empresas que desejam optar por novas tecnologias e aderir inovações em seus produtos.

Palavras-Chave: Indústria 4.0; P\&D; Desenvolvimento de produtos.
\end{abstract}

\begin{abstract}
The search for innovation and new market technologies has grown over the years, causing the emergence of the 4th Industrial Revolution, characterized by Industry 4.0. Therefore, there is a need for investment, by companies and large organizations, in areas related to the product development process (PDP), which consists of a business process that formalizes activities for the development of products and services. The organization of this process helps in improving product quality and reducing costs, making the company more agile and competitive. The article aims to map, analyze, and explore the flows that occur in the process of developing new products in a laboratory of an educational institution. As methodology, the qualitative, descriptive and exploratory research system was applied, based on this study. With the following study, it was possible to understand the need to formalize, map and understand the process of understanding and comparing theory. It can be concluded that the P\&D sector has been gaining space in the market, and the investment in the development process new products is extremely important for each and every organization, and the full knowledge generates opportunities for improvement for organization that wishes to opt for new technologies and adhere to innovations in their products.

Keywords: Industry 4.0; P\&D; Product's development
\end{abstract}

\section{Introdução}

Existem três Revoluções Industriais que influenciaram nas mudanças dos processos produtivos ao decorrer dos anos. Atualmente, observamos uma quarta ganhando cada vez mais força em todo mundo, chamada de Indústria 4.0 que vem sendo estudada e investigada antes e durante o período de sua ocorrência.

A Indústria 4.0 prevê a integração entre humanos e máquinas, mesmo que em posições geográficas distantes, formando grandes redes e fornecendo produtos e serviços de forma autônoma (Silva; Santos Filho; Miyagi, 2015). Dessa forma, a Indústria 4.0 agrega valor

Afiliação dos autores:

${ }^{1}$ Universidade de Vassouras - UV, Vassouras, RJ, Brasil. 
à toda estrutura organizacional, afetando diversos níveis dos processos produtivos, como por exemplo o projeto, as operações, os produtos e até mesmo a manufatura, ou seja, toda cadeia de suprimentos.

Em uma economia cada vez mais globalizada, as empresas buscam satisfazer as necessidades e exigências dos consumidores para se manterem competitivas no mercado.Acompetitividade de mercado está diretamente ligada à capacidade empresarial de se manter à frente no mercado em relação às outras organizações. Dessa forma, torna-se essencial introduzir novos produtos ao mercado, seja com conteúdo tecnológico, novas características de qualidade, desempenho, custo, entre outros.

Segundo Takahashi \& Takahashi (2007), o desenvolvimento de produto é um dos principais agentes no que diz respeito a agregar valor ao mercado. É resultante da necessidade do cliente, tornando então o abstrato - ideias e conceitos - ao real. Na literatura são propostas diversas metodologias para o desenvolvimento de novos produtos, cabendo às empresas encontrar ou adequar aquela que melhor se adapte a sua realidade e cultura.

Entretanto, a dificuldade, normalmente, está em encontrar profissionais capazes de gerenciar esse processo, com características sistêmicas, que envolvem diversas áreas da organização, como planejamento e desenvolvimento de produtos, marketing, engenharia e produção, tornando-se oportuno o estudo em questão.

\section{Indústria 4.0}

Com o passar dos anos, a evolução tecnológica tem sido um fator de grande importância e de grande responsabilidade pelos avanços das produções, sejam elas fabris ou dadas como prestação de serviços. Tal avanço se dá pela otimização do tempo e pela precisão de execução de tarefas relacionadas à produção.

Histórica e conceitualmente falando, a primeira revolução industrial se deu no ano de 1780 com o marcante uso do motor a vapor em fábricas, o que resultou na centralização dos processos fabris. A segunda revolução industrial teve início na segunda metade do século XIX, na qual ficaram marcados diversos desenvolvimentos nos ramos da indústria química, elétrica, de aço e de petróleo. Falando da terceira revolução industrial, que ocorreu no final do ano 1960, também conhecida como revolução técnico-científica e informacional, foi uma revolução tecnológica propriamente dita, pois os marcos dessa fase foram os avanços da informática, das telecomunicações, da robótica e da nanotecnologia (Drath; Horch, 2014).

O termo Indústria 4.0 surgiu na Alemanha no ano de 2011, sendo referência do que se tornaria a quarta revolução industrial (Drath; Horch, 2014), tendo como foco a melhoria contínua da produtividade dos processos industriais e empresariais.

A indústria 4.0 está diretamente ligada às áreas de tecnologias como a internet das coisas, a robótica avançada e os sistemas de conexão máquina-máquina que possibilita a interação das máquinas entre si nos processos de produção para que haja maiores detalhes e precisão na confecção dos produtos e sem perder a possibilidade de produção em grande escala (Lasi et al., 2014).

A integração e o trabalho unificado entre humanos e máquinas são o que a indústria 4.0 prevê, mesmo que haja, geograficamente, uma grande distância entre eles, é possível realizar tarefas por meio de grandes redes de interações tecnológicas (Silva; Santos Filho; Miyagi, 2015). Os sistemas cyber-físicos estão diretamente ligados a essa indústria, pois é por meio deles que os equipamentos conectados por meio da internet das coisas conseguem trocar informações em tempo real para tomarem decisões autônomas (Kagermann; Wahlster; Helbig, 2013; Lasi et al.,2014).

Diversas áreas de formação estudam a indústria 4.0, como a engenharia, a administração, a ciência da computação, a geografia, entre outros (Lasi et al., 2014). Pois se trata de uma tecnologia capaz de informatizar e otimizar não somente a área de atuação fabril, mas como qualquer outra área de produção ou de gerenciamento de dados.

\section{A competitividade de mercado}

Em uma economia cada vez mais globalizada e com os avanços tecnológicos impostos pelo cenário da Indústria 4.0, as organizações buscam satisfazer as necessidades e exigências dos consumidores, e assim, manterem-se competitivas no mercado onde atuam. Uma organização que se diz competitiva, segundo Fernandes (2003, p 65), deve direcionar suas ações para ter o controle de todas as tecnologias chaves e ter conhecimento de no mínimo uma das tecnologias em crescimento dentro do setor.

A condição de líder de mercado é alcançada quando determinada empresa além de conseguir gerar faturamento, se torna referência de boa qualidade e padrão entre os clientes. A competitividade gerada entre as empresas, por um lado, pode gerar dificuldades e dúvidas, entretanto, por outro lado gera também motivação aos colaboradores a sempre buscarem especialização e melhorias em relação aos concorrentes.

De acordo com Porter (1999, p.84) a constante revolução da tecnologia da informação está interferindo na competitividade de três modos consideráveis, 
que são: alterar as regras da competição de acordo com a mudança de estrutura setorial; gerar vantagem competitiva ao estabelecer novas maneiras de alcançar e superar a atuação dos rivais; e disseminar operações totalmente novas a partir das operações presentes da organização.

Alguns dos fatores que podem melhorar bastante a competitividade além das motivações ditas, são: o capital humano, a gestão otimizada, a gestão da inovação e a tecnologia. Segundo Porter (1986, p. 175), a inovação tecnológica é a principal fonte de sucesso de uma mudança estrutural, de acordo com o autor, o que pode gerar ampliação de mercado e com isso gerar também um crescimento da empresa é a inovação referente ao produto ofertado aos clientes. Porém, tudo deve ser devidamente planejado e organizado, pois tal inovação também pode gerar, indiretamente, alguns obstáculos relacionados à mobilidade e a novos métodos de produção, que irão afetar consequentemente as economias de escala da organização.

Toda e qualquer organização necessita saber lidar com a pressão de um mercado competitivo, para assim sempre querer superar, além das outras empresas, os próprios resultados a fim de obter uma melhoria contínua. Fleury \& Oliveira Jr. (2001), partem da definição de que os gerentes acreditam corretamente que os investimentos direcionados à tecnologia de informação impulsionam as habilidades e motivações não somente das pessoas que prestam serviços para a empresa, mas também aqueles que trabalham para a organização fora dela. O estímulo das empresas é de grande valia para a consolidação da liderança de mercado, já que com os colaboradores motivados as chances de um aumento de faturamento e de vendas crescem, além de maior fidelização dos clientes e concretização no mercado.

O caminho para uma empresa se tornar líder de mercado não é simples e muito menos fácil, depende diretamente de muito esforço no trabalho e muito conhecimento e tempo destinados ao objetivo. Um dos primeiros passos a serem dados é descobrir o que realmente o público-alvo quer receber como produto ou serviço da organização, para assim trabalhar em cima dessa informação para entregar ao cliente um trabalho cada vez melhor e manter um bom relacionamento com o público. Albrecht (1994) diz que o que constitui o resultado da aprovação ou não do cliente é a percepção que se tem da satisfação ou não satisfação de uma necessidade em questão, e essa questão pode ter grande abrangência, desde uma pequena peça tangível até uma experiência do cliente propriamente dita.

Em relação a como se destacar no mercado em relação às demais empresas, primordialmente deve-se dar devida atenção ao planejamento estratégico, pois é de suma importância de que todos os fatores capacitantes a levarem a empresa a um nível de liderança sejam considerados, desde a gestão interna de pessoas até o setor de marketing diretamente ligado ao público tem de haver uma harmonia e definições de objetivos e modos de trabalho.

\section{Projeto de Desenvolvimento de Produtos}

Atualmente, num cenário de grande competitividade no mercado, onde a concorrência é cada vez maior, segundo Brown \& Esisenhardt a internacionalização das operações e rápidas mudanças tecnológicas, exigem das empresas agilidade, produtividade e alta qualidade, características que dependem diretamente da efetividade do Processo de Desenvolvimento de Produto (PDP).

A origem do processo formal de desenvolvimento de produtos ocorreu no século XIX, e, a partir das Revoluções Industriais, os conhecimentos adquiridos no PDP foram sofrendo alterações, incluindo a estrutura hierárquica desse processo. Surgem, então, propostas inéditas de modelos referenciais de PDP durante o final do século XX, objetivando o esclarecimento e facilitação do processo, junto com a sistematização em modelos de referência, que o representam.

Um dos guias utilizados por muitos autores como modelo referencial é o proposto por Rozenfeld (2006). Considerado como modelo mais completo, ele contempla a divisão do processo em três macrofases que são: pré-desenvolvimento, desenvolvimento e pósdesenvolvimento, e apresenta, definidas explicitamente, as atividades de geração e acompanhamento dos requisitos ao longo do $\mathrm{PDP}$.

Amacrofase de pré-desenvolvimento compreende duas etapas, o plano estratégico de negócios que consiste no plano estratégico do negócio em si, a minuta do projeto, bem como o portfólio de produtos. $\mathrm{E}$, o planejamento do projeto engloba todo escopo do projeto e do produto, decisão do cronograma, avaliação de riscos, análise de viabilidade econômica do projeto, até o planejamento do projeto do produto.

Já a macrofase de desenvolvimento abrange cinco fases, primeiro o projeto informacional que analisa e define as necessidades do cliente, os requisitos e metas do produto e seu ciclo de vida. Em segundo, o projeto conceitual que engloba as definições do produto como sua função global, os materiais que serão utilizados, o layout, macroprocesso de fabricação, lista inicial de Sistema, Subsistemas e Componentes (SSC), entre outros. A terceira fase é o projeto detalhado, na qual ocorrem o detalhamento dos SSCs, protótipo funcional, planejamento dos processos de fabricação, montagem, planejamento do fim de vida do produto, projeto aprovado para desenvolvimento com o produto autorizado.

A quarta etapa, preparação da produção do 
produto, que engloba todo recebimento e instalação dos recursos da produção, produção do lote piloto, homologação do processo, otimização da produção e certificação do produto, treinamento dos funcionários e todo planejamento do marketing. Por fim, a quinta fase, que seria o lançamento do produto, no qual ocorre todo desenvolvimento do processo de vendas, distribuição, atendimento ao cliente, assistência técnica, marketing de lançamento, até o lançamento do produto no mercado.

A macrofase de pós-desenvolvimento contém duas fases, o acompanhamento do produto em que as funções principais são a análise dos relatórios de desempenho do produto, as propostas de necessidades de modificações no produto ou serviço relacionado, desempenho econômico, síntese das lições aprendidas, até a decisão de descontinuidade do produto e enfim, a fase de descontinuidade do produto que consiste em todo o plano de descontinuidade e relatório de retirada do produto do mercado.

Em resumo, as etapas para modelo de desenvolvimento de produto definem-se por:

- Etapa 1: Geração do conceito: (1.1) Geração de ideias; (1.2) Especificação de oportunidades.

- Etapa 2: Projeto preliminar.

- Etapa 3: Projeto detalhado e protótipo.

- Etapa 4: Definição do custo e processo de produção: (4.1) Composição do custo do produto/ serviço; (4.2) Descrição do processo de produção (layout); (4.3) Fluxo do processo/equipamento/mão-deobra.

- Etapa 5: Transformando Ideias em negócios: (5.1) Exploração da oportunidade; (5.2) Lógica do negócio.

Apesar da importância dos modelos expostos, convém ressaltar que os autores enfatizam que os modelos devem estar adequados às próprias necessidades da empresa, de modo a facilitar a gestão e a entrega das partes importantes dos projetos.

\section{Pesquisa e Desenvolvimento (P\&D)}

A globalização da economia ocasionou grandes modificações nas estruturas organizacionais das empresas, tornando a sobrevivência um fator dependente do desenvolvimento de melhorias contínuas e da própria inovação (Martins et al., 2019).

As empresas estão voltadas para a busca incessante do lucro a partir da redução de custos e aumento da produção. Porém, para Berto e Dias Junior (2015), obter qualidade e produtividade está diretamente relacionado ao investimento em pesquisa e desenvolvimento.

Em outras palavras, a área de P\&D é responsável pela realização de pesquisas como principal metodologia para levantar informações a respeito das necessidades dos clientes e através dos resultados realizar melhorias nos processos produtivos, em paralelo com o desenvolvimento de produtos, permitindo que as empresas criem novas fontes de receita e cresçam no mercado. Ou seja, a P\&D deixou de ser uma opção e tornou-se uma necessidade para as empresas de modo geral, pois a cultura de inovação institucionalizouse, gerando maior desempenho e crescimento aos negócios.

As atividades de P\&D tem a característica de ser a mais tradicional das atividades de inovação, destacando-se pela influência exercida no processo de inovação tecnológica das empresas (Martins, 2019). Em síntese ao mencionado é possível destacar a importância das atividades de pesquisa e desenvolvimento para as organizações que desejam se manter competitivas no mercado onde atuam e se perpetuar como precursoras da inovação.

Com isso, convém ponderar que o desenvolvimento de produtos está diretamente ligado às atividades de $\mathrm{P} \& \mathrm{D}$ e se fazem amplamente presentes no cotidiano das organizações.

\section{Design Thinking e Brainstorming}

Segundo Santos et al., (2017), a evolução do mercado tecnológico e a globalização impulsionou a necessidade da busca pela inovação, a fim de adaptar-se à nova ótica de gestão empresarial. Na opinião de Rocha (2009), é de suma importância que os empresários e gestores - antes da concorrência - empenhem-se no que diz respeito à entenderem as tendências de mercado.

Diante disso, o uso de ferramentas aplicadas à gestão empresarial, tomada de decisões e solução de problemas, torna-se imprescindível a toda e qualquer organização que busca seu espaço no mercado. No setor de $\mathrm{P} \& \mathrm{D}$, por exemplo, são utilizadas técnicas como o Design Thinking e Brainstorming a fim de mapear o produto e/ou serviço para assim suprir uma necessidade e/ou resolver um problema.

Pela visão de Cooper, Junginger e Lockwood (2010), o Design Thinkingé uma ferramenta que contribui no que diz respeito à projeção futura, idealização através de processos e, ainda, no desenvolvimento e criação de produtos e/ou serviços. Segundo Manzini (2008), o profissional da área tem papel importante no que diz respeito ao desenvolvimento estratégico. Para o autor:

"Seu papel específico [...] é oferecer novas soluções
a problemas, sejam velhos ou novos, e propor seus
cenários como tema em processos de discussão social,
colaborando na construção de visões compartilhadas
sobre futuros possíveis (Manzini, 2008, p. 16)."

Segundo Osborn (1987) a definição de brainstorm é "usar o cérebro para tumultuar o problema". O método 
é aplicado à fase de planejamento de um processo e associa-se à criatividade, buscando resoluções para um problema. Para Mazzoti (2018), a finalidade da técnica é de captar ideias de um determinado assunto relacionado a várias competências e áreas de atuação e sem limite máximo de sugestões. As propostas são ouvidas e anotadas, até que formulem uma solução eficiente.

A visão de Mazzoti (2018) traz a concepção de que uma grande seleção de ideias se torna eficaz no que diz respeito à elaboração de novos produtos e/ou soluções para um determinado problema. Com isso, o Brainstorming é considerado um método de extrema eficácia diretamente ligado à idealização de novos projetos e protótipos.

\section{Materiais e Métodos}

Este trabalho utiliza a estratégia de pesquisa qualitativa, descritiva e exploratória, com base em um estudo de caso. Para Gil (2010), o estudo de caso é um método caracterizado pela análise aprofundada e flexível de um ou poucos assuntos, com o objetivo principal de atingir alto grau de detalhamento na pesquisa.

A pesquisa qualitativa se caracteriza por ser uma metodologia exploratória, não-estruturada, que proporciona insights e compreensão do contexto do problema (Malhotra, 2001). Nesse nível, a pesquisa qualitativa envolve uma abordagem interpretativa, ou seja, seus pesquisadores estudam os cenários tentando entender os fenômenos e os significados que as pessoas a eles conferem. E, dessa forma, com um conjunto de práticas materiais e interpretativas dar visibilidade ao mundo através de uma série de representações como as notas de campo, as entrevistas, as fotografias, as gravações, entre outros.

Inicialmentefoirealizadaumarevisãobibliográfica em que as plataformas mais utilizadas foram o Google Acadêmico e a Biblioteca Virtual disponibilizada pela universidade. As palavras utilizadas para a busca foram, principalmente, desenvolvimento de novos produtos e indústria 4.0.

Com o resultado das pesquisas, utilizou-se artigos e livros com o conteúdo de etapas de desenvolvimento de produtos para a realização do estudo com o objetivo de criar um roteiro que seria utilizado para observar como o laboratório realizava a gestão dos processos de desenvolvimento. Dessa forma, foram definidas as seguintes etapas para o roteiro:

- Observar as técnicas utilizadas na etapa de geração de ideias;

- Observar as técnicas utilizadas na etapa de seleção de ideias;

- Observar como eles identificaram as necessidades do cliente;
- Observar as ferramentas utilizadas para identificar as vantagens competitivas do produto;

- Observar de que maneira é avaliado a viabilidade do produto a ser produzido;

- Observar as etapas de fabricação do produto.

Para a escolha do local, foi analisado na instituição de ensino quais eram os laboratórios existentes e se alguns deles eram voltados para o desenvolvimento de novos produtos. Observou-se um número alto de patentes que envolviam um dos laboratórios da instituição, e, dessa forma, foi escolhido onde aplicar a pesquisa em questão.

Com o roteiro em mãos, foi preparada uma visita in loco no laboratório escolhido para observar todas as etapas realizadas durante o processo de criação de um produto. Durante a visita, utilizou-se o roteiro como auxílio para observação, bem como para as anotações realizadas durante a visita para, posteriormente, conectar toda a análise do processo do laboratório com o estudo bibliográfico realizado anteriormente. Em resumo, realizamos o fluxo abaixo com os métodos utilizados (Figura 1).

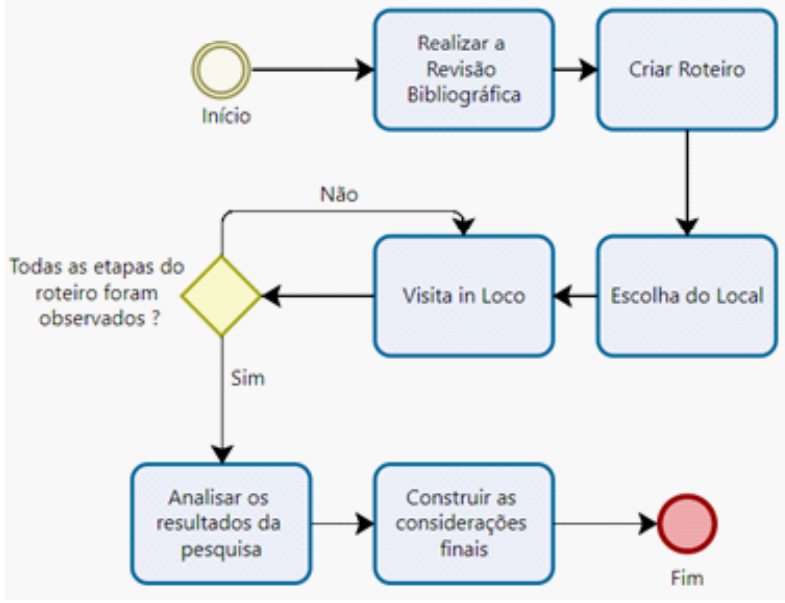

Figura 1. Fluxo dos métodos utilizados para a pesquisa.

Fonte: Autores, 2021.

\section{Resultados}

O laboratório estudado atua na instituição de ensino desde o ano 2019. Atualmente, ele conta com quatro professores que lecionam nos mais diversos cursos da instituição. O laboratório se tornou referência na parte de desenvolvimento de produtos e até agora acumula um número significativo de patentes, com 
aproximadamente dezessete patentes registradas no Instituto Nacional da Propriedade Industrial (INPI), além de, aproximadamente, dez processos em andamento.

A missão do laboratório é, principalmente, oferecer aos alunos de pós-graduação da instituição a possibilidade de criar produtos que impactam em sua área de atuação. $O$ processo de desenvolvimento de produtos no local em questão é impulsionado pelos avanços de natureza tecnológica já presentes no conceito de Indústria 4.0, já que o processo de desenvolvimento do protótipo do produto se dá através da impressão 3D.

A partir de um modelo de referência genérico, uma empresa pode definir um específico, que se torna seu manual de procedimentos e serve de base para especificação de projetos de desenvolvimento de produtos Rozenfeld (2006).

Levando em conta o modelo referencial de Rozenfeld (2006), o PDP do laboratório estudado contempla somente duas macrofases, isto é, não existe formalizada a fase de pós-desenvolvimento. A Figura 3 ilustra as fases do PDP do laboratório, observa-se que o pré-desenvolvimento está baseado nas fases de planejamento do projeto e projeto informacional. E o

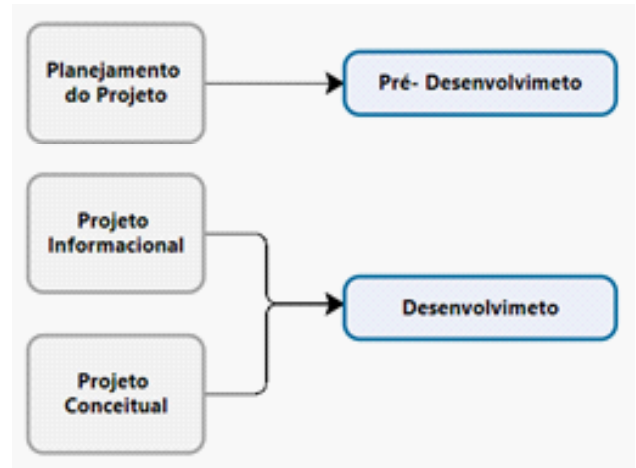

Figura 2. Fases do PDP do laboratório estudado.

Fonte: Autores, 2021.

desenvolvimento compreende basicamente a fase do projeto conceitual.

Na elaboração do modelo utilizado considerou-se as várias metodologias descritas na literatura. Portanto, para complementar o levantamento das informações adquiridas durante a observação, utilizou-se como referência uma parte do modelo de desenvolvimento de produtos com às seguintes etapas:

- Etapa 1: Geração do conceito: (1.1) Geração de ideias; (1.2) Especificação de oportunidades.

- Etapa 2: Projeto preliminar.

- Etapa 3: Projeto detalhado e protótipo.

A primeira etapa trata-se da geração do conceito do produto, dividindo-se nas principais atividades: geração de ideias e especificação de oportunidades. A geração do conceito caracteriza-se como a ideia inicial, onde ocorrem reuniões com o intuito de ajustar e melhorar as concepções conceituais do projeto.

Uma das particularidades do laboratório é que normalmente o aluno, antes da reunião, já tem um produto em mente, portanto o objetivo principal das reuniões é definir o conceito do produto, de que forma ele impacta a sociedade e durante o brainstorming quais são as melhores opções para o produto em questão, ou seja, como será o design, o material que deve ser utilizado, entre outros.

Posteriormente, na fase de geração de ideias, ainda motivada por reuniões, utiliza-se ferramentas como o Design Thinking e Brainstorming, agregando à ideia conceitual as propostas de melhoria citadas e analisadas e/ou implementos, moldando-se cada vez mais próximo ao produto final. Observou-se que no laboratório estudado, eram realizadas reuniões entre os professores responsáveis e o estudante da pós-graduação.

O Design Thinking objetiva-se em direcionar uma soma de soluções construídas às necessidades levantadas anteriormente, caracterizando-se também como "inteligência competitiva". A ferramenta Brainstorming, por sua vez, diz respeito ao processo criativo e de geração de ideias a fim de solucionar um problema e/ou atender uma necessidade específica.

E, por último, a fase de especificações de oportunidades enquadra-se em um pré-requisito no qual avaliam-se quais os tipos de problemas o produto poderá resolver. Feito isso, relata-se, detalhadamente, os problemas e seus respectivos nichos, verificandose através de listagens os possíveis pontos fortes do produto.

Concomitante à visão de Sousa (2015), a segunda etapa faz alusão à tomada de decisão referente ao produto - de forma cuidadosa e prudente - de modo que, as definições anteriores sobre o novo produto em questão não ocasionem potenciais problemas dificultando suas reversões nas próximas etapas do desenvolvimento.

Por fim, a terceira - e última - etapa, simultânea à perspectiva de Souza (2015), o produto é analisado levando em consideração sua forma geométrica e seu layout para assim, posteriormente, realizar a seleção dos materiais necessários para a elaboração do projeto. É importante destacar que nesse ponto é necessário o detalhamento do produto, no que diz respeito à fabricação, montagem, embalagem e, ainda, sua avaliação ergonômica.

Com isso, é realizada a prototipação do produto em uma impressora 3D de acordo com as informações levantadas durante o detalhamento. É válido ressaltar que esse produto não é levado para o mercado, sendo assim, não é comercializado, sua finalidade é voltada para a área acadêmica e profissional para fins de estudos 
teóricos e práticos do laboratório.

Diante das informações apresentadas durante o estudo, observou-se a necessidade de formalizar, mapear e analisar o processo de desenvolvimento de novos produtos, com a finalidade de reduzir, por exemplo, o tempo gasto, os materiais utilizados e, ainda, o custo final do produto.

\section{Discussão}

A globalização da economia e os avanços tecnológicos ocasionaram grandes modificações nas estruturas organizacionais das empresas, tornando a sobrevivência um fator dependente de melhorias contínuas e da própria inovação.

Dessa forma, o PDP, apesar de complexo, é um processo chave para que as empresas mantenham a competitividade exigida pelo mercado, ou seja, formalizar as etapas do processo garante a qualidade do produto.

Neste artigo, utilizou-se como foco o laboratório de pesquisa e desenvolvimento de uma determinada instituição de ensino. A partir das notas de campo feitas durante a visita in loco, procuramos estudar as atividades do local, com o objetivo de mapear o processo durante o desenvolvimento de um novo produto com base nos modelos referenciais desenvolvidos por Rozenfeld (2006).

Por fim, verificou-se que a adoção de um modelo referencial auxilia na gestão do ciclo de desenvolvimento de produtos, além de contribuir para inovações e êxito dos produtos no mercado.

\section{Referências}

Albrecht, K. (1994). Programando o futuro: o trem da linha norte. São Paulo: Mackron Books.

Cooper, R., Junginger, S., \& Lockwood, T. (2009). Design thinking and design management: A research and practice perspective. Design Management Review, 20(2), 46-55.

Drath, R., \& Horch, A. (2014). Industrie 4.0: Hit or hype? [industry forum]. IEEE industrial electronics magazine, 8(2), 56-58.

Fernandes, J. M. (2003) Gestão da tecnologia como parte da estratégia competitiva das empresas. - $1^{\text {a }}$ ed. Brasília: IPDE.

Fleury, M. T. \& Oliveira Jr. M. M. (2001). Gestão estratégica do conhecimento: integrando aprendizagem, conhecimento e competências. São Paulo: Atlas.

Gil, A. C. (2010). Como elaborar projetos de pesquisa (5. ed.). São Paulo: Atlas.

Kagermann, H. et al. (2013) Securing the future of German manufacturing industry: Recommendations for implementing the strategic initiative Industrie 4.0. Frankfurt.

Lasi, H. et al. (2014). Industry 4.0. Business \& Information Systems Engineering, Springer, v. 6, n.4, p. 239-242.

Malhotra, N. K. (2001). Pesquisa de Marketing: uma orientação aplicada. 3. ed. Porto Alegre: Bookman.
Manzini, E. (2008) Design para a inovação social e sustentabilidade: Comunidades criativas, organizações colaborativas e novas redes projetuais. Rio de Janeiro: E-PAPERS.

Martins. et al. (2019). Evidenciação dos investimentos em P\&D e os reflexos no desempenho organizacional de empresas nos países do BRICS. Desenvolve Revista de Gestão do Unilasalle, v.8. n.1.p.83-97.

Mazzoti. et al. (2018). A exploração da criatividade, através do uso da técnica de brainstorming, adaptada ao processo de criação em moda. Revista Artes Moda Design - Novas Fronteiras, Novos Territórios da Arte, do Design e da Moda v. 2 n. 2. Recuperado em 16 de setembro de 2021 de https://www. revistas.udesc.br/index.php/ensinarmode/article/view/11980.

Osborn, A. (1987). O Poder Criador da Mente: principios e processos do pensamento criador e do "brainstorming". Traduzido por E. Jacy Monteiro. São Paulo: Ibrasa editora.

Porter, M. E. (1999). Cluster e competitividade. HSM Manegement. HSM do Brasil ISSN 1415-8868; n. 15, julho-agosto.

Porter, M. E. (1986). Estratégia competitiva. Técnicas para análise de indústrias e da concorrência. $17^{\mathrm{a}}$ ed. Rio de Janeiro: Campus.

Rocha, L. C. (2009). Criatividade e Inovação: Como adaptar-se às mudanças. LTC: Rio de Janeiro.

Rozenfeld, H. et al. (2006). Gestão de desenvolvimento de produtos: uma referência para melhoria do processo. São Paulo: Editora Saraiva.

Santos, P. et al. (2017). A Metodologia Design Thinking Como Estratégia Gerencial Para Empreendimentos. Revista Latino-Americana de Inovação e Engenharia de Produção, v. 5. n.8. p. 25-43, 2017. Recuperado em 16 de setembro de 2021 https://revistas.ufpr.br/relainep/article/view/55490.

Silva, R. M. da. et al. (2015). Modelagem de Sistema de Controle da Indústria 4.0 Baseada em Holon, Agente, Rede de Petri e Arquitetura Orientada a Serviços. In: XII Simpósio Brasileiro de Automação Inteligente. Natal.

Souza, M. et al. (2015). Projeto e desenvolvimento de um novo produto: anilha de encher. XXXV ENCONTRO NACIONAL DE ENGENHARIA DE PRODUÇÃO - ENEGEP. Fortaleza, CE. 13 a 16. outubro. 2015. Recuperado em 16 de setembro de 2021 de http://www.abepro.org.br/biblioteca/TN STO 210 247_27663.pdf.

Takayashi, S. \& Takayashi, V. P. (2007). Gestão de inovação de produtos: estratégia, processo, organização e conhecimento. Rio de Janeiro: Editora Campus. 